Discrete Comput Geom 35:117-129 (2006)

DOI: $10.1007 / \mathrm{s} 00454-005-1182-2$

\title{
Tropical Secant Varieties of Linear Spaces
}

\author{
Mike Develin \\ American Institute of Mathematics, 360 Portage Avenue, \\ Palo Alto, CA 94306-2244, USA \\ develin@post.harvard.edu
}

\begin{abstract}
In this paper we investigate tropical secant varieties of ordinary linear spaces. These correspond to the log-limit sets of ordinary toric varieties; we show that their interesting parts are combinatorially isomorphic to a certain natural subcomplex of the complex of regular subdivisions of a corresponding point set, and we display the range of behavior of this object. We also use this characterization to reformulate the question of determining Barvinok rank into a question regarding regular subdivisions of products of simplices.
\end{abstract}

\section{Introduction}

The tropical semiring is given by the real numbers $\mathbb{R}$, together with the operations of tropical addition $\oplus$ given by $a \oplus b=\min (a, b)$ and tropical multiplication $\otimes$ given by $a \otimes b=a+b$. As in ordinary geometry, we consider the tropical semimodule $\mathbb{R}^{d+1}$, as well as the corresponding tropical projective space $\mathbb{T P}^{d}=\mathbb{R}^{d+1} /(1, \ldots, 1)$ given by modding out by tropical scalar multiplication. There has been a recent spate of work in tropical geometry, as well as the use of tropical geometry to solve problems arising in ordinary geometry. In particular, theories of tropical convexity [4], tropical polytopes [5], tropical linear spaces [8], tropical linear algebra [3], tropical geometry [7], and tropical algebraic geometry [6] have all been burgeoning.

In this paper, we consider the $k$ th tropical secant variety of an ordinary linear subspace $L$ in tropical projective space $\mathbb{T} \mathbb{P}^{d}$, defined by

$$
S^{k}(L):=\left\{v_{1} \oplus v_{2} \oplus \cdots \oplus v_{k+1} \mid x_{i} \in L\right\} .
$$

In other words, $S^{k}(L)$ consists of points which are the coordinatewise minima of $k+1$ points in $L$. By working in tropical projective space, which is more natural for the applications described below, we assume that $L \subset \mathbb{R}^{d+1}$ contains the vector $(1, \ldots, 1)$; then all tropical secant varieties of $L$ do also, and we mod out by this vector to work in $\mathbb{T P}^{d}$. 
These tropical secant varieties correspond to the log-limits of ordinary toric varieties. To be precise, the image of an ordinary toric variety in the variables $x_{1}, \ldots, x_{n}$ under the logarithm map is a linear space in the variables $\log x_{1}, \ldots, \log x_{n}$; the dimension of the linear space is the same as the dimension of the toric variety. For $x_{i}$ and $y_{i}$ large, we have $\log \left(x_{i}+y_{i}\right) \sim \max \left(\log x_{i}+\log y_{i}\right)$, and so the log-limit of the ordinary secant variety corresponds to the tropical secant variety (the max-plus and min-plus semirings are isomorphic).

Another application of tropical secant varieties of linear subspaces is to the Barvinok subcomplex. A matrix of size $d \times n$ has Barvinok rank at most $k$ if it is expressible as the tropical sum of $k$ tropically rank-one matrices, where a tropically rank-one matrix $M$ is one satisfying $m_{i j}+m_{k l}=m_{i l}+m_{j k}$ for all $i, j, k, l$ [3]. These tropically rank-one matrices form an ordinary linear subspace, and the matrices of Barvinok rank at most $k$ comprise the $k$ th secant variety of this linear subspace. A consequence of our work is that the interesting component of this space is a subcomplex of the secondary polytope of the product of simplices $\Delta_{d-1} \times \Delta_{n-1}$; we also use our results to provide an intuitive algorithm for determining Barvinok rank.

Our aim is to develop a general theory of these tropical secant varieties of ordinary linear spaces. In Section 2 we present and prove our main theorem, which states that the interesting parts of tropical secant varieties of a linear space, which we call tropical secant complexes, are certain natural subcomplexes of the complex of regular subdivisions of a corresponding point configuration. In Section 3 we use this representation to prove that the $k$ th secant variety of any generic line in $d$-space is equal to the cone from a line over the complex of lower faces of the cyclic polytope $C(2 k, d-1)$. In Section 4 we compute the first secant variety of a diverse set of two-dimensional examples, including an example where the corresponding complex is not pure and one where it is not contractible. In Section 5 we apply our theory to the case of $d \times n$ matrices of Barvinok rank $k$, showing that these complexes are certain subcomplexes of the secondary polytope of $\Delta_{d-1} \times \Delta_{n-1}$. A corollary of this is that the complex of matrices of Barvinok rank two is pure.

\section{Tropical Secant Complexes}

In this section we prove the following main theorem allowing us to express tropical secant varieties of linear spaces as subcomplexes of the fan of regular subdivisions of an associated point configuration.

Theorem 2.1. Let $L \subset \mathbb{T} \mathbb{P}^{n-1}$ be the (ordinary) linear subspace of dimension d generated by the $d$ rows of the associated matrix $M_{L}$. Let $V_{L}=\left\{v_{1}, \ldots, v_{n}\right\}$ be the n-point configuration in $\mathbb{R}^{d}$ given by the columns of $M_{L}$. Then a vector $x=\left(x_{1}, \ldots, x_{n}\right)$ is in the $k$ th tropical secant variety of $L$ if and only if the upper envelope of the polytope formed by the height vector $x$ has $k+1$ facets whose union contains each point of $V_{L}$.

Proof. Let the rows of $M_{L}$, the generators of $L$, be denoted by $L_{1}, \ldots, L_{d}$, and let the corresponding coordinates of $V_{L}$ be $z_{1}, \ldots, z_{d}$. A point $x$ is in the $k$ th secant variety of $L$ if and only if there exist points $y^{1}, \ldots, y^{k+1}$ in $L \oplus(1, \ldots, 1) \mathbb{R}$ such that $x_{i}=\min _{j}\left(y_{i}^{j}\right)$. Consider any point $y^{j} \in L$. This is some linear combination of the rows of $L$, plus a 
constant: $y^{j}=a+\sum_{i=1}^{d} c_{i} L_{i}$. The corresponding height function on the points $v_{i}$ is given simply by $f^{j}=a+\sum c_{i} z_{i}$; in other words, the heights given by $y^{j}$ to $V_{L}$ are given by the value of some affine functional on $\mathbb{R}^{d}$ at those points.

Therefore, a point $x$ is in the $k$ th secant variety if there exist $k+1$ affine functionals on $\mathbb{R}^{d}$ whose pointwise minima at the points of $V_{L}$ give the coordinates of $x$. Now, consider the regular subdivision of $V_{L}$ given by the upper envelope of the height vector $x$ as in the statement of the theorem. If these $k+1$ affine functionals exist, then each one defines an upper face of this polytope, since for each functional $f^{j}$ and each point $v_{i}$ we have $x_{i} \leq f^{j}\left(v_{i}\right)$ (since $x_{i}$ is the coordinatewise minimum of the $f^{j}$. Furthermore, for each $v_{i}, x_{i}$ is equal to some $f^{j}\left(v_{i}\right)$, and so $v_{i}$ is in the face defined by $f^{j}$. Therefore, these faces together contain each point of $V_{L}$, and thus so do a set of $k+1$ facets containing them.

Conversely, suppose that the height vector $x$ induces a regular subdivision with $k+1$ upper facets $F_{1}, \ldots, F_{k+1}$ such that each $v_{i}$ is contained in one of them. Each facet $F_{j}$ is contained in some hyperplane $H_{j}$ in the lifted $\mathbb{R}^{d}$ with height vectors, and as before this hyperplane corresponds to an affine functional on $\mathbb{R}^{d}$. If this functional is $a+\sum c_{i} z_{i}$, then define the point $y^{j}$ via $y^{j}=a+\sum_{i=1}^{d} c_{i} L_{i}$. We claim that $y^{1} \oplus \cdots \oplus y^{k+1}=x$. We need to check that they agree in each coordinate, which is the same thing as saying that the height vector $x$ is the coordinatewise minimum of the height vectors $y^{j}$. However, since $F_{j}$ is an upper facet for each $j$, for each $i$ we have $x_{i} \leq y_{i}^{j}$, and since $x_{i}$ is contained in one of the facets, equality is achieved for some $j$. Therefore, $x$ is in the $k$ th secant variety of $L$ as desired.

It is worth noting here that we picked an arbitrary basis for $L$. However, picking a different basis yields an affinely isomorphic point configuration, so as must be the case we can pick any basis to fill out the matrix $M_{L}$.

Since each regular subdivision corresponds to a polyhedral cone of height vectors, Theorem 2.1 gives us a decomposition of the tropical secant variety.

Corollary 2.2. The $k$ th tropical secant variety of a linear subspace $L$ is a cone from $L$ over a polytopal complex, which we call the $k$ th tropical secant complex of $L$. The faces of this polytopal complex correspond to regular subdivisions of $L$ in which there exist $k+1$ facets containing all of the points, with a face $F$ containing a face $G$ if the regular subdivision associated to $F$ refines the one associated to $G$.

Proof. First, consider the case of a regular subdivision not using all of the vertices of the point configuration. In order for a height vector to be valid under Theorem 2.1, the lift of any unused point $x$ must lie on the lifted face whose interior contains $x$, and in particular the height of $x$ is uniquely specified by the vertices of the point configuration, with the space of height vectors inducing that regular subdivision being affinely isomorphic to the space of height vectors on the used vertices inducing the regular subdivision on that point subconfiguration.

This space is clearly a polyhedral cone, with inequalities on the heights given by the set of upper facets; in other words, the inequalities are given by picking a vertex, picking an upper facet, and noting that the point's height must be less than that of the corresponding facet-defining hyperplane. Setting one of these inequalities to an equality 
corresponds to the case where a point off a facet is moved onto the facet, which has the effect of coarsening the regular subdivision, so these cones fit together as stated in the corollary. The original cone is in the $k$ th secant variety if and only if the corresponding regular subdivision has $k+1$ facets whose union is all of the points; this property is preserved under coarsening, so every face of any cone in the $k$ th secant variety is also in the $k$ th secant variety. Therefore, the $k$ th secant variety is a valid polyhedral complex.

To check that it is a cone from $L$ over a polytopal complex, we need first to check for each cone that if $x \in L$, then $x$ is a cone point. However, as before, the height vector of $x \in L$ is simply an affine functional on the space containing the configuration $V_{L}$. Taking a nontrivial linear combination of $x$ with any height vector $y$ simply performs an affine transformation on the heights, which does not change the induced regular subdivision and thus does not change the cone of the height vector as desired.

The other step in checking that each cone is a cone from $L$ of a polytope is to check that the only lineality in each cone is in fact $L$. Indeed, consider any vector $y \notin L$. Because $y$ is not in $L$, it is not an affine function on the configuration $V_{L}$, so $y$ does not induce the trivial subdivision. It immediately follows that $y$ and $-y$ induce different subdivisions, indeed subdivisions with no common refinement. However, given any $x$ in the cone, for large enough $c, x+c y$ refines $y$ and $x-c y$ refines $-y$, so it is impossible that these two are the same regular subdivision, and hence they cannot both be in the original cone. Therefore, $y$ cannot be in the lineality space of the cone as desired.

In the convex case, this gives us something very akin to the secondary polytope. Given a polytope $P$, the secondary polytope [9] is a polytope whose face poset is the poset of regular subdivisions, ordered by refinement, so that the vertices of $P$ are the regular triangulations. If $V_{L}$ is in convex position, and $k$ is large, all regular subdivisions satisfy the condition of Corollary 2.2. The condition for face inclusion is dual to the inclusion in the secondary polytope, so the $k$ th secant variety is a cone from $L$ over the dual of the secondary polytope. In particular, it will be all of $\mathbb{R}^{n}$ in this case. Indeed, this is an if and only if.

Corollary 2.3. The $\infty$ th secant variety of a linear subspace $L$ is all of $\mathbb{R}^{n}$ if and only if the corresponding point configuration $V_{L} \subset \mathbb{R}^{d}$ is in convex position, i.e., if every point of $V_{L}$ is a vertex of $\operatorname{conv}\left(V_{L}\right)$.

Proof. A point $x$ is in the $\infty$ th secant variety if and only if its height vector corresponds to a lift of $V_{L}$ where the union of all of the upper facets contains all of the lifted points. If $V_{L}$ is in convex position, every height vector will have this property, since every point will be in some upper facet. If not, then some point can be written as an affine combination of the other points, $v_{i}=\sum_{j \neq i} c_{j} v_{j}$ with $\sum c_{j}=1$. Then if $x_{i}<\sum_{j \neq i} c_{j} x_{j}$, the lifted point $v_{i}$ will not be in the upper envelope of the convex hull of all of the lifted points, and so it will be in no upper facet. Therefore, any $x$ satisfying this condition will not be in the $\infty$ th secant variety of $L$. 
We can easily compute the dimension of the $k$ th tropical secant variety in the case where the linear subspace is generic in the sense that the point configuration $V_{L}$ is in general position, i.e. has no $d+1$ points lying in an affine subspace of dimension $d-1$.

Proposition 2.4. Suppose that the d-dimensional linear subspace $L$ is generic. Then the $k$ th secant variety of $L$ is a complex of dimension $\min ((k+1)(d+1)-1, n)$.

Proof. By Corollary 2.2, the faces of the $k$ th tropical secant variety of $x$ correspond to regular subdivisions of the $(n+1)$-point configuration $V_{L}$ in $\mathbb{R}^{d}$ in which there exist $k+1$ facets whose union contains all of the points. The condition of the theorem implies that $V_{L}$ consists of $n+1$ distinct points. Since the tropical secant variety is a subset of $\mathbb{T} \mathbb{P}^{n}$, it obviously must have dimension at most $n$.

First, suppose that $(k+1)(d+1)-1 \leq n$. Pick a generic linear functional; this orders the points in $V_{L}$ in some order, $v_{1}, \ldots, v_{n+1}$. Then by examining the values of this linear functional at these points, it is evident that the $d$-polytopes formed by the convex hulls of the sets

$$
\begin{array}{r}
\left\{v_{1}, \ldots, v_{d+1}\right\},\left\{v_{d+2}, \ldots, v_{2 d+2}\right\}, \ldots,\left\{v_{1+(k-1) d}, \ldots,\right. \\
\left.v_{1+(k-1)(d+1)-1}\right\}, \\
\\
\left\{v_{1+(k-1)(d+1)}, \ldots, v_{n}\right\}
\end{array}
$$

do not intersect. These are $k d$-simplices and one facet which is not a simplex (they have dimension $d$ since the point configuration $V_{L}$ is in general position). Since these facets do not intersect, there exists a regular subdivision containing all of them. Take a regular subdivision from this nonempty set which is as fine as possible. We claim that the corresponding cell of the $k$ th tropical secant variety has dimension $(k+1)(d+1)-1$. Indeed, consider a height vector in its relative interior. For each of the $k+1 d$-polytopes in our set, we have $d+1$ degrees of freedom for the heights corresponding to those vertices, which gives us $(k+1)(d+1)$ degrees of freedom which we can move in without changing the subdivision. Furthermore, in the relative interior of this cell, wiggling these points can only refine the rest of the induced subdivision, and since the cell was chosen to be maximal with respect to refinement, it also leaves the remainder of the subdivision fixed. Therefore, the cell of height vectors has dimension equal to $(k+1)(d+1)$, which upon projectivization yields a cell of dimension $(k+1)(d+1)-1$ in the $k$ th tropical secant variety.

On the other hand, any cell of the $k$ th tropical secant variety corresponds to a regular subdivision with $k+1$ facets whose union is all of $V_{L}$. Each of these facets is $d$ dimensional, and so in order for a height vector to be in this cell, the coordinates of the height vector which correspond to each facet must lie in a $(d+1)$-dimensional space. Since each coordinate corresponds to at least one facet, we have at most $(k+1)(d+1)$ degrees of freedom in the cell, and so the maximal dimension of any cell in the tropical secant variety is in fact $(k+1)(d+1)-1$ as desired.

Finally, suppose $(k+1)(d+1)-1 \geq n$. We need to prove that there exists some cell of dimension $n$ in the $k$ th tropical secant variety of $L$. As before, we find a linear functional, which orders the points $v_{1}, \ldots, v_{n+1}$. For our (at most) $k+1$ facets, we take as many $(d+1)$ 's as possible, until we are left with some remainder less than $d+1$; for the final facet, we take a simplex including this leftover subset and some points from the 
last set of $d+1$, and as in the first case we find a triangulation including these facets and as fine as possible given that constraint. Then by the same logic as before, it immediately follows that we have $n$ degrees of freedom which we can move in without changing the subdivision in question.

This maximal dimension is what one would expect; given some generic $d$-dimensional shape in ordinary space, the $k$ th secant variety consists of the union of all points in $k$ planes spanned by $k+1$ points from the shape. The number of degrees of freedom of this space should be as follows: for each of the $k+1$ points, we have $d$ degrees of freedom, and we have a final $k$ degrees of freedom for picking the point once the plane is fixed, for a total of $(k+1) d+k=(k+1)(d+1)-1$, just as in the tropical secant case. The first tropical secant variety is especially nice.

Proposition 2.5. If the $d$-dimensional linear space $L$ is generic, then the first tropical secant variety of $L$ is a pure complex.

Proof. By Proposition 2.4, the dimension of the first tropical secant variety of $L$ is $\min (2 d+1, n)$. We need to show that every regular subdivision which contains two facets whose union contains each point in $V_{L}$ can be refined to one with this many degrees of freedom. First, suppose $2 d+1<n$. If our two facets do not intersect, then as in the proof of the previous proposition, we take a regular subdivision containing the provided one which is as fine as possible. The corresponding cell will then have $d+1$ degrees of freedom for each facet for a total of $2 d+1$ (after projectivization.)

If the two facets do not intersect, by the previous case, it suffices to show that this complex can be refined to one with two nonintersecting spanning facets. Suppose they intersect in an $r$-face; we will induct on $r$. Since $V_{L}$ is in general position, this face must be a simplex with $r+1$ points. Since $n>2 d+1$, and we have $n+r+1$ points with multiplicity, one of the two facets must have at least $d+2$ points. Take one overlap point out of it; the remaining points form a full-dimensional convex hull. Then we can refine the previous subdivision by breaking this facet; rigorously, what we are doing is lowering the height of the shared vertex by an infinitesimal amount and adjusting the heights of facet $F_{1}$ so that the lifted points still share a hyperplane. This process has the effect of taking the shared vertex out of the upper facet-defining hyperplane of $F_{2}$; the new, refined subdivision (which may be strictly finer in other places as well) refines the original one and has two facets whose union is all of $V_{L}$ overlapping in a face of smaller dimension. By induction, we are finished.

Next, suppose $2 d+1 \geq n$. We need to check that every valid regular subdivision can be refined to one whose cell has dimension $n$. If the two spanning facets are both simplices, then we can refine this regular subdivision to a regular triangulation, whose cell has $n$ degrees of freedom. If not, then as in the first case we can remove one of the overlap points from one facet, eventually reducing to the case where both facets are simplices. This completes the proof of the proposition.

However, the second tropical secant variety (or complex) is not pure even for points in general position, and the first tropical secant variety is not pure if we do not assume that 
the points are in general position. We give examples of these deviant cases, as well as an example of a nongeneric linear subspace where the dimension is wrong, in Section 4.

\section{The One-Dimensional Case}

In this section we apply the results of Section 2 to the one-dimensional case, i.e., when $L$ is a line in $\mathbb{T P}^{n}$. This corresponds to the case of a (projective) toric curve in $n$-space. In this case we can completely compute the $k$ th tropical secant complex.

Theorem 3.1. Let $L$ be a line in $\mathbb{T} \mathbb{P}^{n}$, generated by $\left(r_{1}, \ldots, r_{n+1}\right)$. Then the $k$ th secant complex of $L$ consists of the set of lower faces of the cyclic polytope $C(2 k, d-2)$ (i.e., $d-2$ points in dimension $2 k$ ), where $d$ is the number of distinct elements of $\left\{r_{1}, \ldots, r_{n+1}\right\}$.

Proof. The corresponding point configuration $V_{L}$ is $n+1$ points on the real line, located at $r_{1}, \ldots, r_{n+1}$; we are looking for the space of height vectors $\left(x_{0}, \ldots, x_{n}\right)$ such that the points $\left\{\left(r_{1}, x_{1}\right), \ldots,\left(r_{n+1}, x_{n+1}\right)\right\}$ have $k$ facets in their upper envelope which together contain all the points. To begin with, if any $r_{i}$ and $r_{j}$ are identical, then the corresponding $x_{i}$ and $x_{j}$ must also be, as otherwise whichever is lower will have that point not in the upper envelope at all. This reduces us to the case where the $r_{i}$ are distinct. We also assume that $r_{0}<r_{1}<\cdots<r_{n}$.

A height vector $\left(x_{0}, \ldots, x_{n}\right)$ is completely determined by the value of $x_{0}$ and the slopes $s_{i}=\left(x_{i+1}-x_{i}\right) /\left(r_{i+1}-r_{i}\right)$ for $1 \leq i \leq n$. The condition that all of the points be in the upper envelope reduces to the constraint $s_{i} \leq s_{i-1}$ for $1 \leq i \leq n-1$. When $s_{i}=s_{i-1}$, the points $\left(r_{i-1}, x_{i-1}\right),\left(r_{i}, x_{i}\right)$, and $\left(r_{i+1}, x_{i+1}\right)$ are all in the same facet of the corresponding regular triangulation. We represent the $n$ slopes by points, putting a bar between two points if the corresponding slopes differ; see Fig. 1 for the corresponding illustration.

It is plain to see that the vertices of the complex provided by Corollary 2.2 (for any $k$ ), which are the coarsest nontrivial regular subdivisions, correspond to patterns with just one bar, of which there are $n-1$.

Therefore, to compute the complex of the $k$ th secant variety, it suffices to compute the finest regular subdivisions with $k+1$ facets whose union is all of the points. The

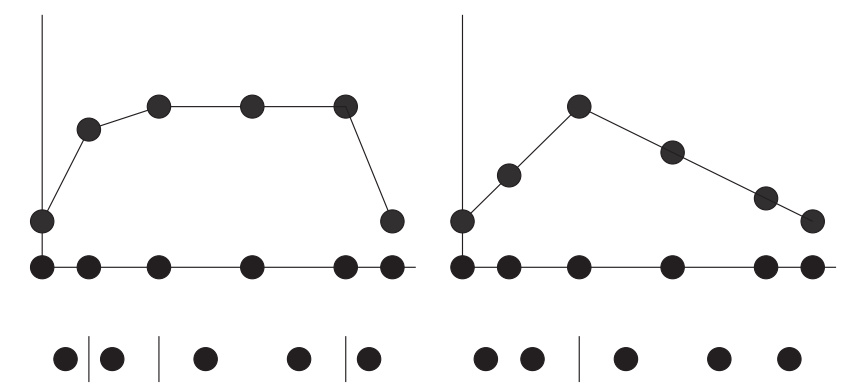

Fig. 1. Construction of bar patterns from height vectors of a one-dimensional point configuration. 
$n+1$ lifted points are represented by the $n+1$ spaces in between the dots (counting the beginning and the end), and the facets of a lifted configuration are given by the closed intervals between adjacent bars.

Suppose we have a bar pattern which is part of the $k$ th secant variety. The $k+1$ facets given by Theorem 2.1 clearly must contain the interval from the first space to the first bar. If the space following the first bar is not filled by a bar, then in order for the union of these facets to contain this space, we must also take the facet consisting of the interval from the first bar to the second bar. If this is the case, then we can insert a bar in the space following the first bar, and take the shortened facet from the new bar to the old second bar along with the other $k$ original facets; we have just demonstrated that this augmented bar pattern still has the property.

Therefore, any bar pattern which corresponds to a facet of the tropical secant complex must have its first two bars adjacent. By identical reasoning, it in fact follows that the bars must come in adjacent pairs; the facets we take consist of every other interval, including the first and last. Counting carefully, we conclude that there must be $k$ pairs of consecutive bars or $2 k$ bars in all.

So, the condition to be a facet of the tropical secant complex is that the included points must come in consecutive pairs. This is precisely the same as Gale's evenness condition [9] for being a facet of the cyclic polytope $C(2 k, n-1)$, except that our condition has the added stipulation that the initial and final segments of bars must have even length. This stipulation is easily seen to correspond exactly to the statement that the facet in question is a lower one, and so the $k$ th tropical secant complex is (combinatorially) isomorphic to the complex of lower faces of the cyclic polytope $C(2 k, n-1)$ as desired.

In the one-dimensional case the $k$ th tropical secant complex is the same regardless of the (generic) linear subspace chosen; this is because the oriented matroid of any $n$-point configuration in general position is the same, and so the space of regular triangulations of these configurations are all combinatorially isomorphic. In the two-dimensional case, corresponding to toric surfaces, the oriented matroid statement is of course no longer true, and this leads to a wide variety of behavior of these tropical secant varieties. We investigate this behavior in the next section.

\section{Examples}

In the previous section we completely computed the tropical secant complexes of all onedimensional linear subspaces. In particular, these $k$ th secant complexes were all pure and contractible. In this section we compute a variety of examples, in which we show that these phenomena were purely low-dimensional: even in two dimensions and even for $k=1$, the phylum of tropical secant complexes is diverse, including species which are not pure and not contractible. We also demonstrate nongeneric examples where the dimension of the tropical secant variety is not in accordance with Proposition 2.4. Rather than give the details of all of the computations we present diagrams of the complexes associated to various configurations (with labeled vertices and facets) as well as pointing out some relevant aspects. 


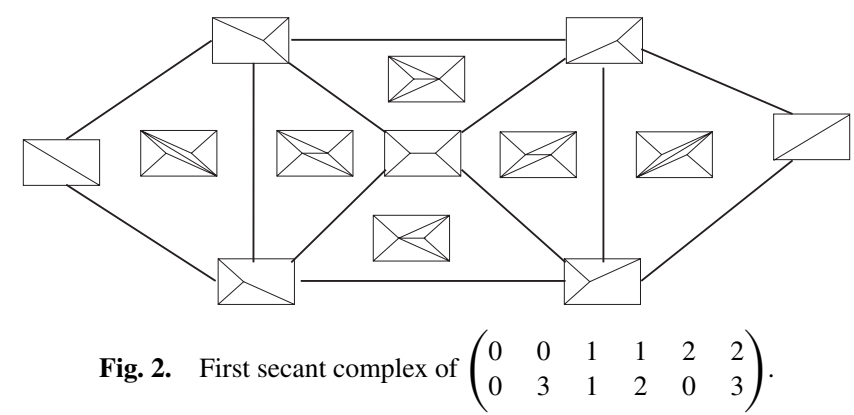

Our first example is a point configuration in general, nonconvex position: a square with two points inside it. The corresponding linear subspace is generated by the rows of the matrix with the points as its columns:

$$
\left(\begin{array}{llllll}
0 & 0 & 1 & 1 & 2 & 2 \\
0 & 3 & 1 & 2 & 0 & 3
\end{array}\right)
$$

This linear subspace, a two-dimensional linear subspace in $\mathbb{T} \mathbb{P}^{5}$, exhibits fairly normal behavior. Its first secant variety is the right dimension (five); indeed, the first secant variety is equal to the $k$ th secant variety for all $k>1$, since every regular subdivision of this point configuration has two facets whose union is the whole space (one containing the left three points and one containing the right three points.) This first tropical secant complex is shown in Fig. 2. Since the points are in general position, it is pure by Proposition 2.5, and it is also contractible.

Our next example is $n$ points in general convex position, i.e., an $n$-gon. For $n=6$, the second secant complex is simply the dual of the secondary polytope of the hexagon, since every regular subdivision contains three facets whose union is all of the points. The first secant complex, however, is interesting: in particular, it is not contractible. For a general $n$-gon, the first secant complex's vertices will correspond to diagonals; the facets (it is a two-dimensional complex) correspond to subdivisions given by three diagonals $\{(a, b+1),(a, b),(a+1, b)\}$. For $n \geq 7$, this complex forms a Möbius strip. For $n=6$, we show the diagram in Fig. 3; this is the first secant complex of the linear subspace generated by the rows of

$$
\left(\begin{array}{llllll}
0 & 0 & 1 & 1 & 2 & 2 \\
0 & 1 & 0 & 2 & 1 & 2
\end{array}\right) .
$$

It is a subcomplex of the dual of the secondary polytope, which has 14 facets corresponding to the 14 triangulations of a hexagon. Six of these have the property that they have two facets whose union contains all of the vertices; these are the six facets in this first secant complex.

It is worth noting that the second secant complex of an $n$-gon is already not pure. Take $n=9$; then the regular subdivision on the left-hand side of Fig. 4 is a face of dimension four in the complex not contained in any face of dimension five, while the subdivision on the right-hand side is a face of dimension five. The spanning facets are shaded.

In our third example the first secant complex is not even pure (Fig. 5). The point configuration $V_{L}$ consists of two sets of three collinear points intersecting at a common 


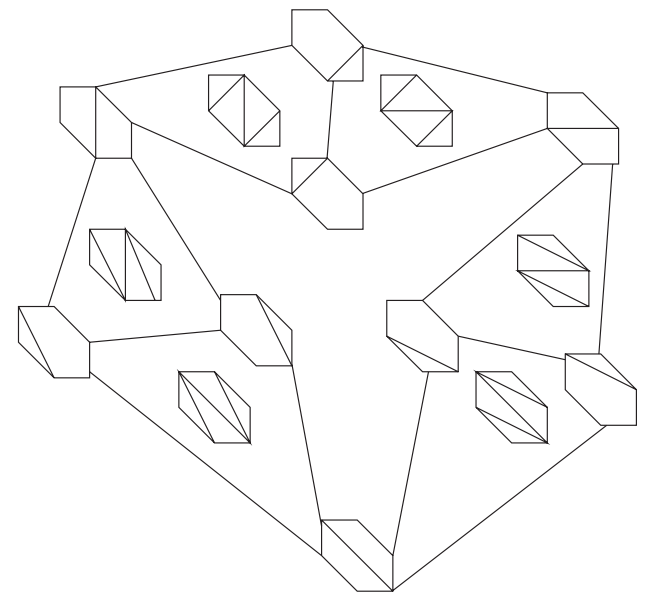

Fig. 3. First secant complex of $\left(\begin{array}{llllll}0 & 0 & 1 & 1 & 2 & 2 \\ 0 & 1 & 0 & 2 & 1 & 2\end{array}\right)$.
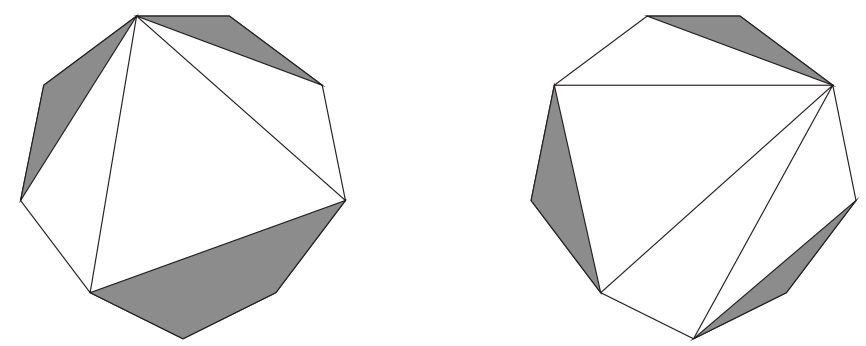

Fig. 4. Regular subdivisions corresponding to maximal faces of different dimension in the second tropical secant complex of the linear subspace corresponding to a nonagon.

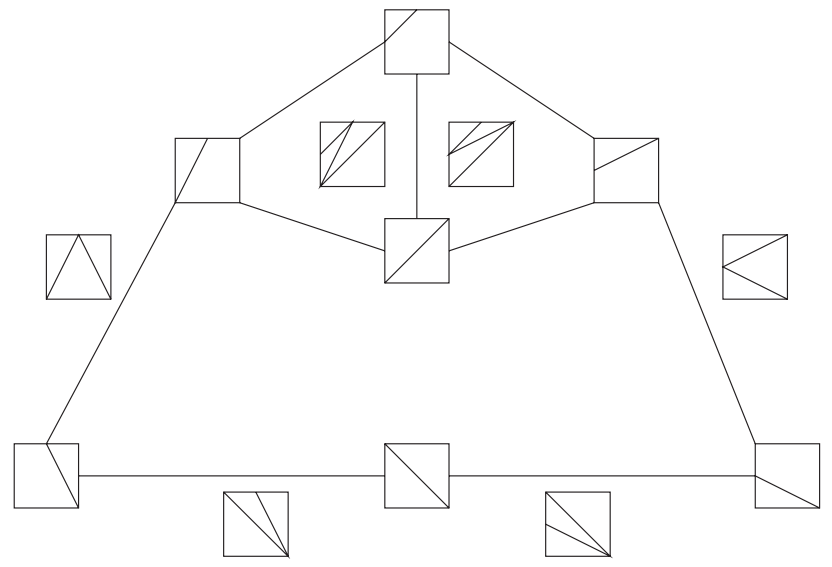

Fig. 5. First secant complex of $\left(\begin{array}{cccccc}0 & 0 & 0 & 1 & 2 & 2 \\ 0 & 1 & 2 & 0 & 0 & 2\end{array}\right)$. 
end point, along with a point in the cone formed by the emanating rays, i.e., the point configuration corresponding to the linear subspace/matrix

$$
\left(\begin{array}{llllll}
0 & 0 & 0 & 1 & 2 & 2 \\
0 & 1 & 2 & 0 & 0 & 2
\end{array}\right)
$$

This complex consists of two triangles along with four edges, a "diamond ring" graph. It is topologically equivalent to a circle and is decidedly impure. We can achieve the same behavior in convex position in one higher dimension by taking a point configuration consisting of a cube with a point beyond a facet. The subdivision given by two opposite square pyramids including the extra point and a triangulation elsewhere is then a maximal face in the complex, but it has lower dimension than another face of the complex, namely the one consisting of the extra point and three points of the facet nearest it, the square pyramid formed by the other five points, and simplices to fill out the remainder of the cube.

In both the second and third examples the property responsible for the impureness is an oriented matroid one. In the maximal secant complex face of inappropriate dimension, two facets of the spanning set overlap, and ordinarily (if the points are in general position) we are able to deal with this by removing a point from one of them, thus refining the subdivision. However, in the deficient cases the overlap point is an isthmus in the matroids of both spanning facets, and thus we cannot remove it from either without degenerating them.

The previous example shows that first secant complexes can be impure, although in those examples they at least have the right dimension. However, this need not be the case, as the next example shows. Here, the point configuration $V_{L}$ consists of one point off a line and five points on a line, corresponding to the linear subspace generated by the rows of

$$
\left(\begin{array}{llllll}
0 & 0 & 0 & 0 & 0 & 2 \\
0 & 1 & 2 & 3 & 4 & 0
\end{array}\right)
$$

Proposition 2.4 tells us that the first tropical secant variety should have dimension $(k+1)(d+1)-1=5$. However, it has only dimension four: that is, the first tropical secant complex is one-dimensional, not two-dimensional. Its vertices correspond to the coarsest possible nontrivial subdivisions, those with one dividing line segment; there are no two-faces, which correspond to having three dividing line segments, since the only regular subdivision with three dividing line segments does not have two facets whose union is the whole space. The first tropical secant complex, which consists of two line segments intersecting at a single point, is shown in Fig. 6.

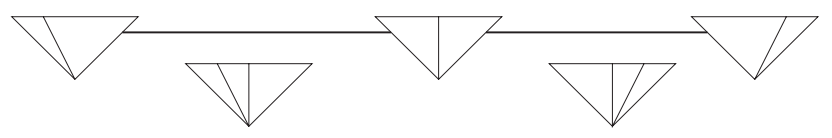

Fig. 6. First secant complex of $\left(\begin{array}{llllll}0 & 0 & 0 & 0 & 0 & 2 \\ 0 & 1 & 2 & 3 & 4 & 0\end{array}\right)$. 
This diversity among tropical secant varieties, even for first secant varieties of twodimensional linear subspaces, is just a peek at the inviting theory. These complexes need not be pure, of the predicted dimension, or contractible. In the next section we shift our focus to applying the theory to an already existing setup, namely the complex of matrices of fixed Barvinok rank.

\section{Barvinok Rank}

The following definition (taken from [3]) is important in combinatorial optimization.

Definition 5.1. A matrix has Barvinok rank $k$ if it can be expressed as the tropical sum of $k$ matrices of tropical rank one, but not as the tropical sum of $k-1$ such matrices. A matrix $M$ has tropical rank one if we can write $M_{i j}=x_{i}+y_{j}$ for some $x_{i}$ 's and $y_{j}$ 's.

Barvinok et al. [1] showed that for matrices of fixed Barvinok rank $k$, the traveling salesman problem can be solved in polynomial time. Thus, an algorithm for finding the Barvinok rank of a matrix, or a description of the space of matrices of Barvinok rank $k$, is important. We have previously considered this problem in papers [3] (with Bernd Sturmfels and Francisco Santos) and [2].

Using the terminology in this paper, an attractive reformulation of the problem emerges. It is evident from the definition that a matrix $M \in \mathbb{R}^{d \times n}$ has Barvinok rank $k$ if it lies in the $(k-1)$ st secant variety of $L$, where $L$ is the space consisting of all matrices of tropical rank one. This is a linear subspace in $d n$ variables, defined by the equations $M_{i j}+M_{k l}=M_{i l}+M_{k j}$. A basis for this linear subspace of $\mathbb{T P}^{n \times d}$ is given by the matrices $R_{1}, \ldots, R_{d-1}, C_{1}, \ldots, C_{n-1}$, where $R_{i}$ has 1 's in the $i$ th row and 0 's everywhere else, and $C_{j}$ has 1 's in the $j$ th column and 0 's everywhere else. The columns of the matrix with these rows are all 0-1 vectors with zero or one 1's among the first $d$ coordinates, and zero or one 1's among the last $n-1$. This point configuration consists of the vertices of the product of simplices $\Delta^{d-1} \times \Delta^{n-1}$.

Therefore, to determine the Barvinok rank of a matrix $M \in \mathbb{R}^{d \times n}$, it suffices to consider the regular triangulation induced by the corresponding height vector on $P=$ $\Delta^{d-1} \times \Delta^{n-1}$, i.e., by $\omega(i, j)=M_{i j}$, where $i \in[d]$ and $j \in[n]$. The Barvinok rank will be the smallest number of facets needed to cover all of the vertices of $P$. Similarly, the complexes of Barvinok rank $2, \ldots, \min (d, n)$ are nested subcomplexes of the secondary polytope of $P$.

\section{References}

1. A. Barvinok, D.S. Johnson, and G.J. Woeginger, The maximum traveling salesman problem under polyhedral norms, in Integer Programming and Combinatorial Optimization, Lecture Notes in Computer Science 1412, Springer, Berlin, 1998, pp. 195-201.

2. M. Develin, The space of $n$ points on a tropical line in $d$-space, preprint, to appear in Collectanea Mathematica, math. CO/O401224.

3. M. Develin, F. Santos, and B. Sturmfels, On the rank of a tropical matrix, preprint, math. CO/0312114.

4. M. Develin and B. Sturmfels, Tropical convexity, Documenta Mathematica 9 (2004), 1-27. 
5. M. Joswig, Tropical half-spaces, preprint, math. CO/0312068.

6. G. Mikhalkin, Enumerative tropical algebraic geometry in $\mathbb{R}^{2}$, preprint, math. AG / 0312530 .

7. J. Richter-Gebert, B. Sturmfels, and T. Theobald, First steps in tropical geometry, to appear in Idempotent Mathematics and Mathematical Physics, math.AG/0306366.

8. D. Speyer and B. Sturmfels, The tropical Grassmannian, Advances in Geometry 3 (2004), 389-411.

9. G. Ziegler, Lectures on Polytopes, Graduate Texts in Mathematics, Springer, New York, 1995.

Received May 7, 2004, and in revised form April 3, 2004. Online publication August 19, 2005. 